\title{
Preoperative thoracic false lumen branches are predictors of aortic enlargement after stent grafting for DeBakey IIIb aortic dissection
}

Feng Liu, MD, Yang Yang Ge, MD, Wei Guo, MD, Xiao Ping Liu, MD, Xin Jia, MD, Jiang Xiong, MD, and

Xiao Hui Ma, MD

\section{ABSTRACT}

Objective: The study objective was to assess the effect of preoperative thoracic false lumen branches on thoracic aortic enlargement along the stent graft after thoracic endovascular aortic repair for DeBakey IIIb aortic dissection.

Methods: From January 2011 to December 2013, 67 patients who underwent thoracic endovascular aortic repair for DeBakey IIIb aortic dissection were retrospectively investigated. We assessed preoperative thoracic false lumen branches and diameter measurements at the level of the tracheal carina. Patients with a median follow-up of 12.2 months (interquartile range, 4.3-26.6 months) were categorized into 2 groups based on a receiver operating characteristic curve analysis: group A (preoperative thoracic false lumen branches $\geq 8, n=28$ ) and group B (preoperative thoracic false lumen branches $<8, \mathrm{n}=39$ ). Diameter changes over time were evaluated using a mixed model approach. A multivariate Cox regression analysis was used to study the influence of the preoperative thoracic false lumen branches on thoracic aortic enlargement along the stent graft.

Results: Six patients experienced thoracic aortic enlargement along the stent graft during follow-up (6 in group A, 0 in group B). The cumulative thoracic aortic enlargement along the stent-graft rate was higher in group A than in group B (log-rank $P<.01)$. Diameter data showed significantly more reduction of the thoracic false lumen from preprocedure to 12 months in group B than in group A (change difference, 2.51; standard error, $0.99 ; P=.01$ ). Complete false lumen thrombosis was more prominent in group B than in group A at each follow-up time point. After multivariate adjustment, preoperative thoracic false lumen branches were associated with thoracic aortic enlargement along the stent graft (hazard ratio, 2.92; 95\% confidence interval, 1.06-8.01).

Conclusions: Preoperative thoracic false lumen branches are a morphologic predictor of thoracic aortic enlargement along the stent graft in DeBakey IIIb aortic dissection after thoracic endovascular aortic repair. (J Thorac Cardiovasc Surg 2018;155:21-9)

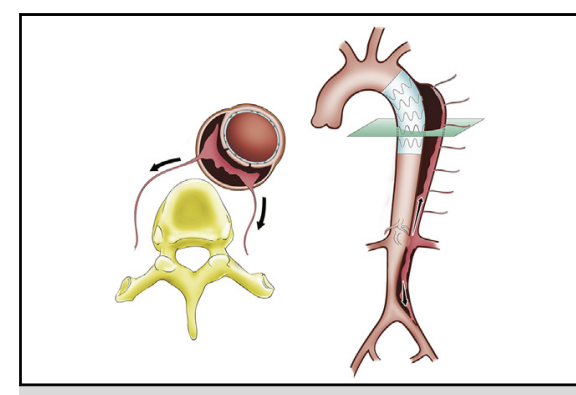

The anatomic basis of continuous perfusion of the thoracic aortic false lumen.

Central Message

Preoperative TFLBs are a predictor of the TAES in DeBakey IIIb aortic dissection after TEVAR.

Perspective

Preoperative TFLB was identified as a prognostic factor for thoracic aortic enlargement after TEVAR in DeBakey IIIb aortic dissection. This may help to identify patients at a higher risk of postoperative thoracic aortic enlargement by imaging scan performed before endovascular therapy.

See Editorial Commentary page 30 .
Thoracic endovascular aortic repair (TEVAR) has been accepted as an effective treatment option for patients with DeBakey III aortic dissections. ${ }^{1-7}$ During the procedure,

\footnotetext{
From the Department of Vascular and Endovascular Surgery, Chinese PLA General Hospital, Beijing, China.

F.L. and Y.Y.G. contributed equally to this work and are co-first authors.

Received for publication Nov 28, 2016; revisions received Aug 24, 2017; accepted for publication Sept 6, 2017; available ahead of print Oct 7, 2017.

Address for reprints: Wei Guo, MD, Department of Endovascular and Vascular Surgery, Chinese PLA General Hospital, 28\# Fuxing Rd, Beijing 100853, China (E-mail: guoweiplagh@sina.com).

$0022-5223 / \$ 36.00$

Copyright (C) 2017 by The American Association for Thoracic Surgery

https://doi.org/10.1016/j.jtcvs.2017.09.010
}

perfusion of the false lumen is eliminated by the closure of the proximal entry tear, and false lumen thrombosis is induced. Eventually, thrombus regression results in aortic remodeling and prevents late enlargement. ${ }^{8-11}$ The most prominent aortic remodeling mainly occurs in

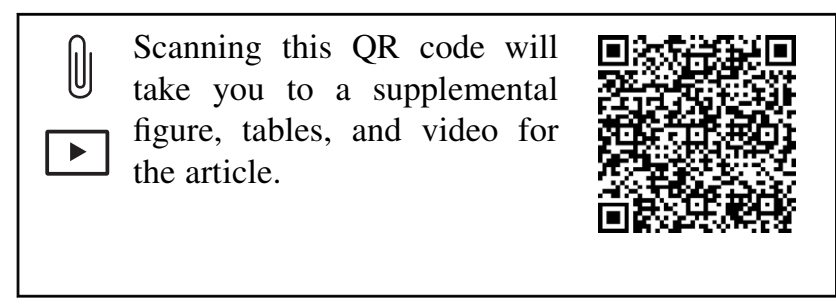




\section{Abbreviations and Acronyms \\ CI = confidence interval \\ CTA $=$ computed tomography angiography \\ HR = hazard ratio \\ $\mathrm{SE} \quad=$ standard error \\ TAES $=$ thoracic aortic enlargement along the stent graft \\ TEVAR $=$ thoracic endovascular aortic repair \\ TFLB $=$ thoracic false lumen branch}

the stent-graft-covered segment. ${ }^{10-12}$ However, a Swedish multicenter study demonstrated that approximately $5 \%$ of patients had thoracic aortic enlargement along the stent graft (TAES) during follow-up. ${ }^{13}$

Persistent false lumen flow is a major factor for unfavorable aortic remodeling. ${ }^{14,15}$ One possible contributing factor for persistent retrograde false lumen flow along the stent-graftcovered segment after TEVAR is abdominal reentry tears. ${ }^{16,17}$ In addition, based on the principle of flow continuity, the outflow has an effect on maintaining the patency of the false lumen. ${ }^{18}$ Kolbel and colleagues ${ }^{19}$ and Song and colleagues ${ }^{20}$ proposed that intercostal and bronchial arteries originating from the thoracic false lumen may play the roles of outflow tracts, leading to chronic aortic dissection, which limits the response to standard TEVAR. However, there is a paucity of clinical data to assess the association between preoperative thoracic false lumen branches (TFLBs) and aortic remodeling along the stent-graft-covered segment.

In this study, we evaluated the association between preoperative TFLBs and postoperative TAES in DeBakey IIIb aortic dissection and investigated the effect of TFLBs on aortic remodeling of the segment along the stent graft after TEVAR.

\section{MATERIALS AND METHODS \\ Study Population}

From January 2011 to December 2013, 245 consecutive patients underwent TEVAR for DeBakey IIIb in our institution. To minimize the potential impact of endoleak on thoracic aortic remodeling, patients with partial or complete left subclavian artery coverage by stent graft $(n=127)$ or with type I endoleak during follow-up $(n=6)$ were excluded. Patients secondary to trauma or connective tissue disease were excluded $(n=26)$. A total of 19 patients were not eligible for the analysis because of missing computed tomography angiography (CTA) data. Ultimately, 67 patients were enrolled in this study. Demographic and clinical information, operative details, and follow-up data were obtained retrospectively. This study was approved by the Institutional Review Board of the Chinese PLA General Hospital, and written informed consent was obtained from all patients.

\section{Thoracic Endovascular Aortic Repair and Adjunctive Procedures}

Fifty patients were treated in the acute setting (defined as $\leq 14$ days from symptom onset), and 17 patients were treated in the chronic setting (defined as $>14$ days from symptom onset). ${ }^{21}$
The indications for TEVAR in the 42 patients with complicated dissections, 33 patients in the acute phase, and 9 patients in the chronic phase were signs of rupture and impending rupture (hemothorax, increasing periaortic, mediastinal hematoma, and pleural effusion), persistent or recurrent pain, hypertension refractory to medical treatment, malperfusion syndrome (acute limb ischemia, bowel ischemia, and renal ischemia), or early enlargement of the false lumen. ${ }^{22}$ Endografting procedure was used routinely for uncomplicated dissections in our center to facilitate aortic remodeling and prevent long-term adverse events; 25 uncomplicated patients underwent TEVAR in total, with 17 in the acute phase and 8 in the chronic phase.

TEVAR and all adjunctive procedures performed in our institution were conducted as previously described. ${ }^{23}$ The following commercially available stent grafts were used: Zenith TX2 (COOK, Bjaeverskov, Denmark) $(\mathrm{n}=16)$; Valiant (Medtronic, Santa Rosa, Calif) $(\mathrm{n}=13)$; Hercules (Microport, Shanghai, China) $(\mathrm{n}=22)$, and Ankura (Lifetech, Shenzhen, China) $(\mathrm{n}=16)$. Adjunctive distal restrictive bare stents were used in 7 patients for the prevention of distal stent-graft-induced new reentry. ${ }^{24}$ The stents were placed in the visceral or iliac arteries when necessary. After discharge, patients were instructed to return to undergo CTA at intervals of 6 and 12 months and annually thereafter. Postoperative arterial- and delayed-phase CTA were performed to assess for false lumen patency and endoleak. ${ }^{25,26}$

\section{Imaging Measurements}

CTA images performed between the time of discharge and May 2014 or death were collected retrospectively. The serials of CTA images available for analysis at $6 \pm 3$ months, $12 \pm 3$ months, and $24 \pm 6$ months were 67 , 45 , and 25 , respectively. The CTA images (slice spacing, 0.6-1.5 mm) were transferred to a 3mensio Workstation (version 6.1; 3mensio Medical Imaging BV, Maastricht, Netherlands) for semiautomatic diameter measurement with a central lumen line method. Two experienced, independent physicians blinded to the clinical information evaluated the morphologic features. Observer variability was estimated by examining the levels of intraobserver and interobserver agreement, according to Bland and Altman, ${ }^{27}$ and the result confirmed small mean differences in terms of its clinical relevance.

The maximum diameter of the entire aorta and the largest short-axial diameter of the true and false lumens were measured at the level of the tracheal carina of the trachea, perpendicular to the central lumen line. ${ }^{28}$ TAES was defined as an increase in maximum aortic diameter greater than $5 \mathrm{~mm}$ at a given follow-up time point compared with the measurement obtained during the preoperative examination. ${ }^{13,29,30}$ Thoracic aortic stabilization was defined as the maximum aortic diameter increasing less than $5 \mathrm{~mm}$, decreasing, or remaining unchanged. The degree of thoracic false lumen thrombosis was classified as patent, partial, or complete. ${ }^{14}$ Preoperatively, measurements were obtained throughout the entire descending thoracic aorta. Postoperatively, these measurements were obtained only in the stent-graft portion of the aorta.

The following specific variables were assessed on the axial slices of preoperative CTA images: the number of TFLBs along the descending thoracic aorta, the number of abdominal false lumen small branches along the abdominal aorta, and the number of visceral branches that arose partially or completely from the false lumen. Preoperative TFLBs were identified in cases in which the false lumen vascularized the following branches: bronchial arteries, mediastinal arteries, esophageal arteries, pericardial arteries, superior phrenic arteries, and posterior intercostal arteries (Figure 1, A-C). The length of dissected aorta and height index were defined as the length of the dissected aorta divided by the body height in an attempt to reduce the impact of body height heterogeneity in individuals. Retrograde type A dissections after TEVAR in our study were symptomatic, and CTA images after onset were excluded.

\section{Statistical Analysis}

Two comparison groups were created on the basis of the results of our time-dependent receiver operating characteristic curve analysis. The 

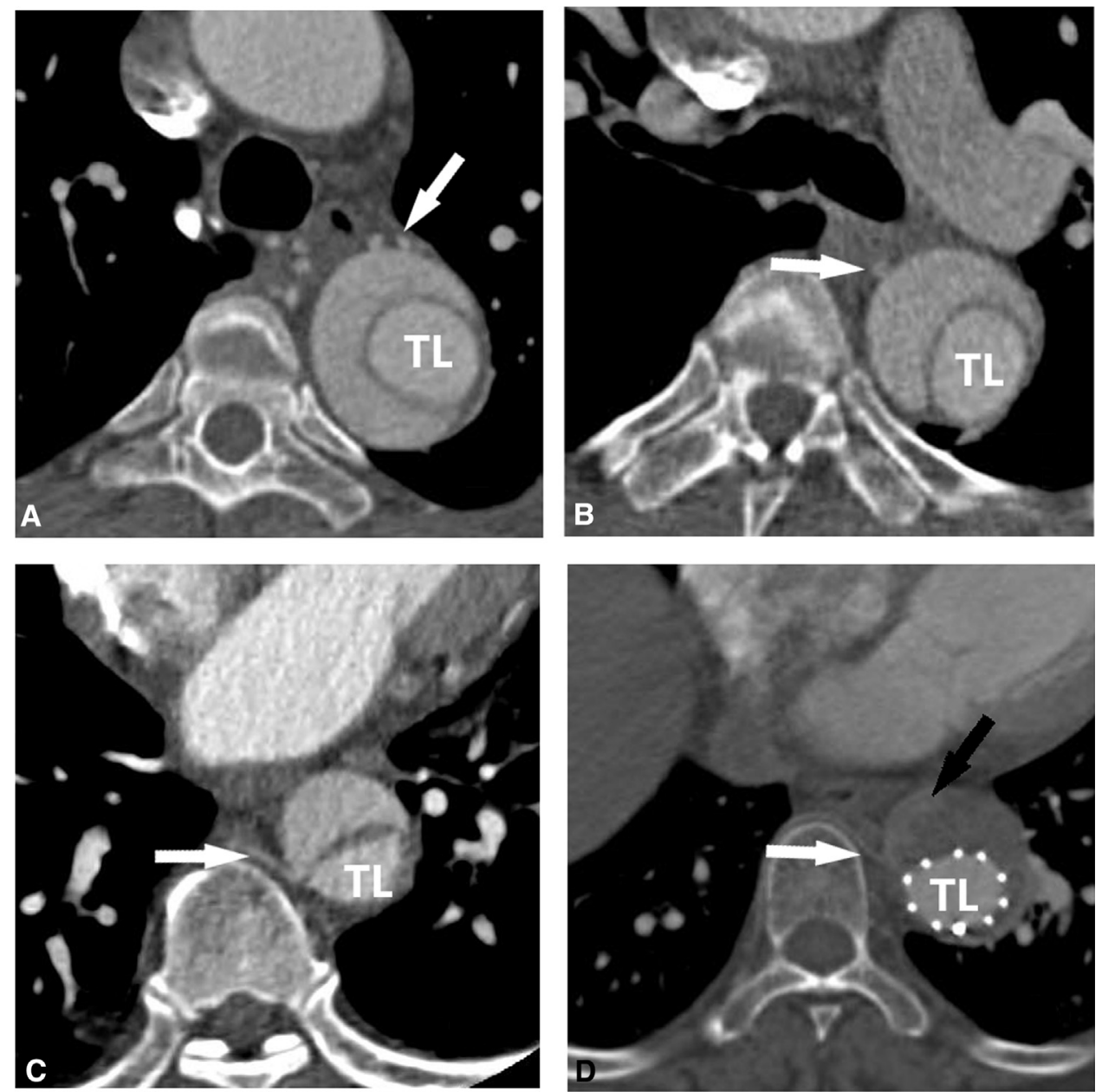

FIGURE 1. Axial image of CTA shows the TFLBs. A, Preoperative left and right bronchial arteries (arrow) arising from the false lumen. B, Preoperative esophageal artery (arrow) arising from false lumen. C, Preoperative posterior intercostal arteries (arrow) arising from false lumen. D, Postoperative posterior intercostal arteries (white arrow) arising from a partially patent false lumen (black arrow). $T L$, True lumen.

receiver operating characteristic curve for TAES had an area under the curve of 0.864 (Figure E1). The number of preoperative TFLBs at an optimal cutoff value of 8 had a high sensitivity $(100 \%)$ and specificity $(69.4 \%)$ for TAES. All the patients were divided into the following 2 groups according to this value: group A (preoperative TFLBs $\geq 8$ ) and group B (preoperative TFLBs $<8$ ). A mixed model approach was used to analyze the changes in aortic diameter over time. Kaplan-Meier analysis was performed to determine the cumulative incidence of TAES

A multivariate Cox proportional hazards regression analysis was performed to investigate the association between preoperative TFLBs and TAES. Covariate identification was based on a $10 \%$ change-in-estimate criterion. ${ }^{31}$ Variables that changed the coefficient of TFLBs by at least $10 \%$ were accepted as covariates for multivariate Cox proportional hazards regression. In addition, covariates were identified on the basis of clinical plausibility and significant association $(P<.05)$ in univariate analysis. To assess the effects of deaths on the estimate of preoperative TFLBs on risk of TAES, sensitivity analyses and competing risk regression were performed. The multivariable competing risk regression was performed, as suggested previously. ${ }^{32,33}$ Statistical calculations were performed using Empower (R) (http://www.empowerstats.com; X\&Y Solutions Inc, Boston, Mass).

\section{RESULTS}

Patients' demographics, clinical features, and dissection characteristics are summarized in Table 1. The mean age of this cohort was $51.2 \pm 10.2$ years, and the median number of preoperative TFLBs was 7 (range, $0-20)$. There was no significant difference with respect to demographic and clinical characteristics between group A (preoperative TFLBs $\geq 8$ ) and group B (preoperative TFLBs $<8$ ). In regard to preoperative morphologic characteristics, group A had a higher number of preoperative abdominal false lumen branches than group B (7 [0-9] vs 4 [0-9], $P=.02$ ). In addition, patent preoperative thoracic false lumens were observed more frequently in group A than in group B $(83.9 \%$ vs $66.7 \%, P=.04)$. Although the limited sample size of the study prevented the differences in these parameters from reaching statistical significance, patients in group A were more likely to have a smaller preoperative true 
TABLE 1. Patients' demographic and clinical characteristics, preoperative morphologic characteristics, and operative outcomes

\begin{tabular}{|c|c|c|c|c|}
\hline Variables & $\begin{array}{l}\text { All patients } \\
(\mathrm{N}=67)\end{array}$ & $\begin{array}{c}\text { Group A } \\
\text { (preoperative TFLBs } \geq 8) \\
(\mathbf{N}=\mathbf{2 8})\end{array}$ & $\begin{array}{c}\text { Group B } \\
\text { (preoperative TFLBs }<8) \\
(\mathbf{N}=\mathbf{3 9})\end{array}$ & $P$ value \\
\hline \multicolumn{5}{|l|}{ Demographics } \\
\hline Male, \% (n) & $85.1(57)$ & $92.9(26)$ & $79.5(31)$ & .17 \\
\hline Age, y & $51.2 \pm 10.2$ & $51.0 \pm 10.8$ & $51.3 \pm 9.8$ & .92 \\
\hline BMI, $\mathrm{kg} / \mathrm{m}^{2}$ & $27.0 \pm 4.2$ & $27.1 \pm 3.9$ & $27.0 \pm 4.5$ & .95 \\
\hline Hypertension & $92.5(62)$ & $92.9(26)$ & $92.3(36)$ & .99 \\
\hline Diabetes & $1.5(1)$ & $0(0)$ & $2.6(1)$ & .99 \\
\hline Hyperlipidemia & $11.9(8)$ & $17.9(5)$ & $7.7(3)$ & .26 \\
\hline Carotid atherosclerosis & $11.9(8)$ & $17.9(5)$ & $7.7(3)$ & .26 \\
\hline Coronary artery disease & $11.9(8)$ & $10.7(3)$ & $12.8(5)$ & .99 \\
\hline Smoking & $52.2(35)$ & $57.1(16)$ & 48.7 (19) & .50 \\
\hline Renal insufficiency & $9.0(6)$ & $10.7(3)$ & $7.7(3)$ & .69 \\
\hline Chronic obstructive pulmonary disease & $4.5(3)$ & $3.6(1)$ & $5.1(2)$ & .99 \\
\hline Phase of aortic dissection, $\%(\mathrm{n})$ & & & & .78 \\
\hline Acute dissection & $74.6(50)$ & $71.4(20)$ & $76.9(30)$ & \\
\hline Chronic dissection & $25.4(17)$ & $28.6(8)$ & $23.1(9)$ & \\
\hline Complicated dissection, $\%$ (n) & $62.7(42)$ & $57.1(16)$ & $66.7(26)$ & .43 \\
\hline \multicolumn{5}{|l|}{ Preoperative morphologic characteristics } \\
\hline No. of TFLBs & $7(0-20)$ & $11(8-20)$ & $4(0-7)$ & $<.01$ \\
\hline Maximum aortic diameter & $37.4 \pm 5.4$ & $37.5 \pm 5.5$ & $37.3 \pm 5.4$ & .88 \\
\hline True lumen diameter & $17.3 \pm 5.4$ & $15.8 \pm 5.1$ & $18.3 \pm 5.4$ & .06 \\
\hline False lumen diameter & $18.5 \pm 6.4$ & $19.9 \pm 6.6$ & $17.5 \pm 6.1$ & .14 \\
\hline $\mathrm{L} / \mathrm{Hi}$ & $0.25 \pm 0.05$ & $0.26 \pm 0.04$ & $0.24 \pm 0.05$ & .06 \\
\hline No. of involved visceral arteries & $1(0-3)$ & $1(0-2)$ & $1(0-3)$ & .34 \\
\hline No. of reentry tears & $1(0-3)$ & $1(0-3)$ & $1(1-2)$ & .15 \\
\hline No. of abdominal false lumen small branches & $5(0-9)$ & $7(0-9)$ & $4(0-9)$ & .02 \\
\hline False lumen status of thoracic aorta, $\%$ (n) & & & & .04 \\
\hline Partially thrombosed & $23.9(16)$ & $10.7(3)$ & $33.3(13)$ & \\
\hline Patent & $76.1(51)$ & $83.9(25)$ & $66.7(26)$ & \\
\hline False lumen status of abdominal aorta, \% (n) & & & & .07 \\
\hline Completely thrombosed & $6.0(4)$ & $0(0)$ & $10.3(4)$ & \\
\hline Partially thrombosed & $14.9(10)$ & $7.1(2)$ & $20.5(8)$ & \\
\hline Patent & $79.1(53)$ & $92.9(26)$ & $69.2(27)$ & \\
\hline Presence fenestration in iliac artery, $\%(n)$ & & & & .09 \\
\hline No & $47.8(32)$ & $35.7(10)$ & $56.4(22)$ & \\
\hline Yes & $52.2(35)$ & $64.3(18)$ & $43.6(17)$ & \\
\hline \multicolumn{5}{|l|}{ Devices implanted } \\
\hline Stent-graft No., \% (n) & & & & 1.00 \\
\hline 1 stent-graft & $95.5(64)$ & $96.4(27)$ & $94.9(37)$ & \\
\hline 2 stent-grafts & $4.5(3)$ & $3.6(1)$ & $5.1(2)$ & \\
\hline Use of restrictive bare stent, $\%(n)$ & $10.4(7)$ & $10.7(3)$ & $10.3(4)$ & 1.00 \\
\hline Additional bare mental stent, $\%$ (n) & $4.5(3)$ & $7.1(2)$ & $2.6(1)$ & .57 \\
\hline Length of stent-graft coverage & $163.3 \pm 27.9$ & $165.9 \pm 28.1$ & $161.5 \pm 28.0$ & .53 \\
\hline
\end{tabular}

Values are mean \pm standard deviation, mean (range), unless otherwise noted. $B M I$, Body mass index; $T F L B$, thoracic false lumen branch; $L / H i$, length of dissected aorta and height index.

lumen diameter $(15.8 \pm 5.1$ vs $18.3 \pm 5.4, P=.06)$, a greater length of dissected aorta and height index $(0.26 \pm 0.04$ vs $0.24 \pm 0.05, P=.06)$, and a higher proportion of patent abdominal false lumens preoperatively $(92.9 \%$ vs $69.2 \%, P=.07)$. Other dissection characteristics, such as indications for TEVAR and devices implanted, were largely similar between the 2 groups.
Because more than $20 \%$ of all patients (19/86) were excluded because of a lack of essential data, comparative analyses were performed between the included and excluded patients. The results of this analysis revealed that there was no significant difference between the 2 cohorts of patients (Table E1), indicating that the patients excluded for missing data may have been random. 


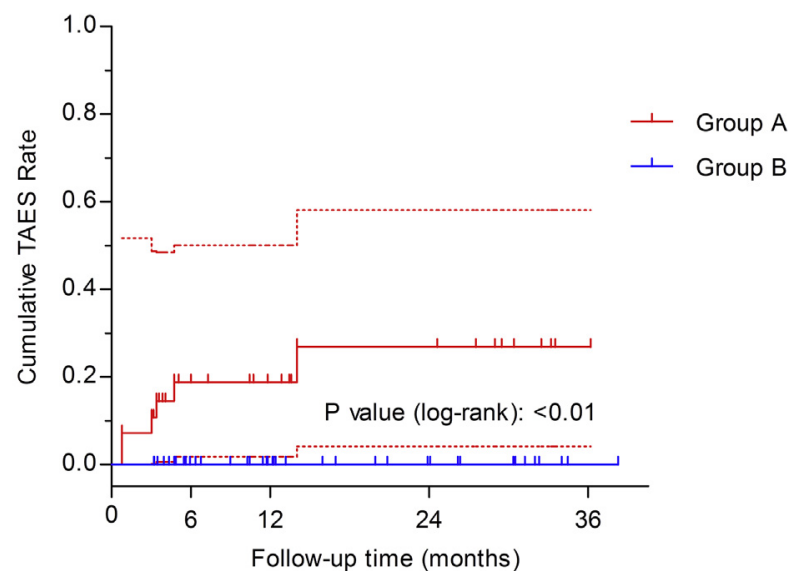

Number at Risk

$\begin{array}{llllll}\text { Group A } & 28 & 18 & 14 & 10 & 2 \\ \text { Group B } & 39 & 29 & 21 & 12\end{array}$

FIGURE 2. Kaplan-Meier analysis of freedom from TAES incidence based on the number of preoperative TFLBs. Group A: TFLBs 8 or greater. Group B: TFLBs less than 8. TAES, Thoracic aortic enlargement along the stent graft.

Six patients experienced TAES during a median imaging follow-up period of 12.2 months (interquartile range, 4.3-26.6 months). All of these patients were in group A. Figure 2 shows the cumulative TAES rates curves for group A and group B. The 12- and 24-month cumulative TAES rates in group A were $18.7 \%$ (95\% confidence interval [CI], 1.72-50.0) and 26.8\% (95\% CI, 4.1-58.0), respectively, versus $0 \%$ and $0 \%$ in group B. The log-rank test showed that the cumulative TAES rate was higher in group A than in group B $(P<.01)$. Among the patients who experienced TAES, 2 deaths occurred in the following months. One occurred at 12 months post-TEVAR due to aortoesophageal fistula, and the other occurred at 19 months post-TEVAR due to retrograde type A dissection. Another 2 patients died of cardiac infarction before the occurrence of TAES

In addition, distal stent-graft-induced new reentry was detected in 7 patients ( 2 in group A vs 5 in group B, $P=.69)$, new reentry in the lower thoracic aorta occurred in 3 patients ( 0 in group A vs 3 in group $\mathrm{B}, P=.26$ ), and retrograde type $A$ dissection occurred in 3 patients ( 2 in group A vs 1 in group $\mathrm{B}, P=.57$ ).

The courses of the changes over time in the maximum diameters of the thoracic aorta, true lumen, and false lumen, stratified according to group A and group B, are shown in Figure 3. From preprocedure to 24 months after the procedure, the maximum thoracic aortic diameter remained stable in group A (change, -0.46 ; standard error [SE], 1.25; $P=.71$ ) but decreased significantly in group B (change, -2.69 ; SE, $0.55 ; P<.01)$. The overall change in the maximum thoracic aortic diameter from preprocedure to

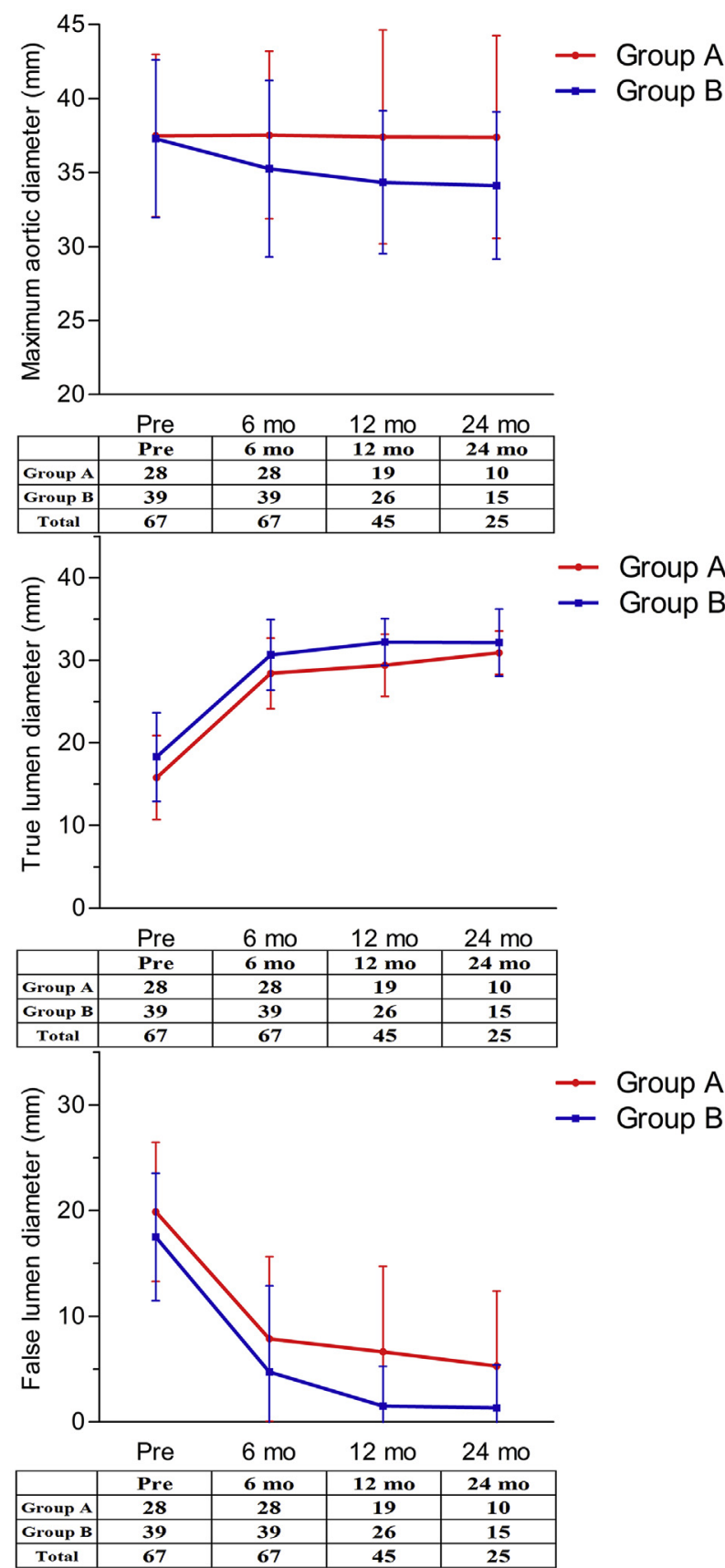

FIGURE 3. Diameter measurement over time.

12 months after the procedure was significantly greater in group B than in group A (change difference, 2.51; SE, $0.99 ; P=.01$ ), but this change was no longer significant 24 months after the procedure (change difference, 2.18; SE, 1.24; $P=.08$ ). Each group exhibited a significant increase in true lumen diameter (group A: change, 14.95; SE, $1.40 ; P<.01$; group $\mathrm{B}$ : change, 14.20 ; SE, 1.12 ; $P<.01)$ and a significant decrease in false lumen diameter (group A: change, $-14.64 ; \mathrm{SE}, 2.30 ; P<.01$; group B: 
TABLE 2. Postoperative false lumen status along the stent-graft-covered segment

\begin{tabular}{|c|c|c|c|c|}
\hline & All patients & $\begin{array}{c}\text { Group A } \\
\text { (preoperative TFLBs } \geq 8)\end{array}$ & $\begin{array}{c}\text { Group B } \\
(\text { Preoperative TFLBs }<8)\end{array}$ & $P$ value \\
\hline Imaging follow-up period, median (IQR) mo $(\mathrm{N})$ & $12.2(4.3-26.6)(67)$ & $11.8(4.5-27.9)(28)$ & $12.2(4.3-25.1)(39)$ & .97 \\
\hline $6 \pm 3 \mathrm{mo}, \%(\mathrm{n} / \mathrm{N})$ & & & & $<.01$ \\
\hline Partially thrombosed & $43.3(29 / 67)$ & $67.9(19 / 28)$ & $25.6(10 / 39)$ & \\
\hline Completely thrombosed & $56.7(38 / 67)$ & $32.1(9 / 28)$ & $74.4(29 / 39)$ & \\
\hline $12 \pm 3 \mathrm{mo}, \%(\mathrm{n} / \mathrm{N})$ & & & & .01 \\
\hline Partially thrombosed & $28.9(13 / 45)$ & $52.6(10 / 19)$ & $11.5(3 / 26)$ & \\
\hline Completely thrombosed & $71.1(32 / 45)$ & $47.4(9 / 19)$ & $88.5(23 / 26)$ & \\
\hline $24 \pm 6 \mathrm{mo}, \%(\mathrm{n} / \mathrm{N})$ & & & & .03 \\
\hline Partially thrombosed & $32.0(8 / 25)$ & $60.0(6 / 10)$ & $13.3(2 / 15)$ & \\
\hline Completely thrombosed & $68.0(17 / 25)$ & $40.0(4 / 10)$ & $86.7(13 / 15)$ & \\
\hline
\end{tabular}

$T F L B$, Thoracic false lumen branch; $I Q R$, interquartile range.

change, -16.23 ; SE, $1.45 ; P<.01)$ from preprocedure to 24 months after the procedure. The overall change in true lumen diameter was not significantly different between group $\mathrm{A}$ and group $\mathrm{B}$, whether from preprocedure to 12 months after the procedure (change difference, -0.51 ; $\mathrm{SE}, 1.42 ; P=.72)$ or from preprocedure to 24 months after the procedure (change difference, $0.76 ; \mathrm{SE}, 1.78 ; P=.67$ ). The overall change in false lumen diameter was significantly different between group A and group B from preprocedure to 12 months after the procedure (change difference, 3.10; SE, 1.45; $P=.03$ ); however, this trend was no longer significant 24 months after the procedure (change difference, 1.58; SE, 1.82; $P=.39$ ).

The postoperative false lumen status along the stentgraft-covered segment at each follow-up time point is shown in Table 2. Complete thrombosis of the false lumen along the stent-graft-covered segment occurred in 56.7\% of all patients at 6 months after the procedure, $71.1 \%$ at 12 months, and $68.0 \%$ at 24 months. Partial thrombosis of the false lumen occurred more frequently in group A than in group B at each follow-up time point (6 months: $67.9 \%$ vs $25.6 \%, P<.01 ; 12$ months: $52.6 \%$ vs $11.5 \%$, $P=.01 ; 24$ months: $60.0 \%$ vs $13.3 \%, P=.03$ ). Furthermore, patent TFLBs delivering retrograde false lumen flow were observed in all patients with partial thrombosis in the false lumen postoperatively.

The univariate Cox regression analyses revealed that no variables were associated with TAES with a $P$ value less than .05 , except for preoperative TFLBs, for which the hazard ratio (HR) was 1.23 (95\% CI, 1.05-1.45), as listed in Table E2. As shown in Table 3, preoperative TFLBs were independently associated with TAES after TEVAR (HR [for 1 increase], 3.22; 95\% CI, 1.22-8.51) after adjustment for gender, hyperlipidemia, and 3 preoperative morphologic variables, including the true lumen diameter, number of involved visceral arteries, and number of abdominal false lumen branches (Table 3, model 2). Further adjustment for the presence of distal stent-graft-induced new reentry during the follow-up period slightly attenuated the association between preoperative TFLBs and TAES (HR [for 1 increase], 2.92; 95\% CI, 1.06-8.01).

To assess the potential bias caused by the 2 deaths before the occurrence of TAES, we performed sensitivity analyses by assuming that the 2 patients who died underwent TAES, as well as competing risk regressions. After adjustment for the aforementioned covariates, the HR was 3.04 (95\% CI, 1.14-8.1) in the final Cox regression model. In the final competing risk regression model, the HR was 1.72 , although the outcome did not reach the statistical significance. These results revealed that the 2 deaths did not materially affect the association between preoperative TFLBs and TAES.

\section{DISCUSSION}

For DeBakey III aortic dissections, the long-term goal of endovascular treatment is to inhibit late dilation of the aorta

TABLE 3. Hazard ratios ( $95 \%$ confidence intervals) for the association of preoperative thoracic false lumen branches with risk of thoracic aortic enlargement along the stent graft

$$
\text { HR }(95 \% \text { CI })
$$

Model 1: nonadjusted Model 2: adjusted for baseline covariates* Model 3: model 2 plus distal SINE

\begin{tabular}{lll}
\hline Preoperative TFLBs (for 1 increase) & $1.23(1.05-1.45)$ & $3.22(1.22-8.51)$ \\
$H R$, Hazard ratio; $C I$, confidence interval; SINE, stent-graft-induced new reentry; $T F L B$, thoracic false lumen branch. *Adjusted for gender, hyperlipidemia, preoperative true
\end{tabular}
lumen diameter, preoperative involved visceral arteries, and preoperative abdominal false lumen branches. †Adjusted for variables in model 1 and distal SINE. 
by promoting aortic remodeling. ${ }^{22}$ Although the most prominent aortic remodeling mainly occurs in the stentgraft-covered segment as opposed to the unstented distal thoracic aorta and the abdominal aorta, several patients have still had enlargement of the stent-graft-covered segment. ${ }^{10-13}$ Our analyses demonstrated that patients with more patent preoperative TFLBs were less likely to develop aortic remodeling than patients with fewer patent preoperative TFLBs, and they potentially had worse clinical outcomes than their counterparts. Preoperative TFLBs are a predictor of the TAES in DeBakey IIIb aortic dissection after TEVAR. After the covariates were held constant, the risk of TAES increased 2.92-fold with a per 1 increase in preoperative TFLBs.

As previously reported, the morphologic characteristics of aortic remodeling include expansion of the true lumen and regression and complete thrombosis of the false lumen. $4,5,8,34,35$ In the current study, the cumulative incidence of TAES was significantly higher among the patients in group A than group B. This result was consistent with the trend in the change in size of the entire aortic lumen: The maximum thoracic aortic diameter decreased significantly over time in group B, but not in group A.

Our results confirmed that the maximum diameters of the thoracic aorta and false lumen tended to decrease more significantly over time in group B than in group A, especially between preprocedure and 12 months after the procedure. The lack of a significant difference in the maximum aortic diameter and false lumen between group A and group B between preprocedure and 24 months after the procedure is probably related to the small number of patients in our series at 24 months after the procedure. Furthermore, by analyzing the changes in size in the true lumen and false lumen between the 2 groups, we could attribute the difference in the maximum aortic diameter changes to the difference in the false lumen diameter changes (Figure 3). These findings suggest that TFLBs can affect the regression of the thoracic aortic false lumen of the stent-graft-covered segment. In addition, patients with more preoperative TFLBs are less likely to benefit from standard TEVAR than patients with fewer preoperative TFLBs.

In our study, complete false lumen thrombosis was more evident in group B than in group A at multiple follow-up time points. As previously reported, persistent perfusion of the false lumen, which hampers complete thrombosis, was associated with aortic dilatation. ${ }^{5,34,36}$ According to our observations, TFLBs functioned as an outflow tract for the perfusion of the stent-graft-covered thoracic aortic false lumen (Video 1). These results further suggest that TFLBs, as outflow tracts of the thoracic false lumen that maintain persistent false lumen perfusion, have a negative impact on the thrombogenic environment.

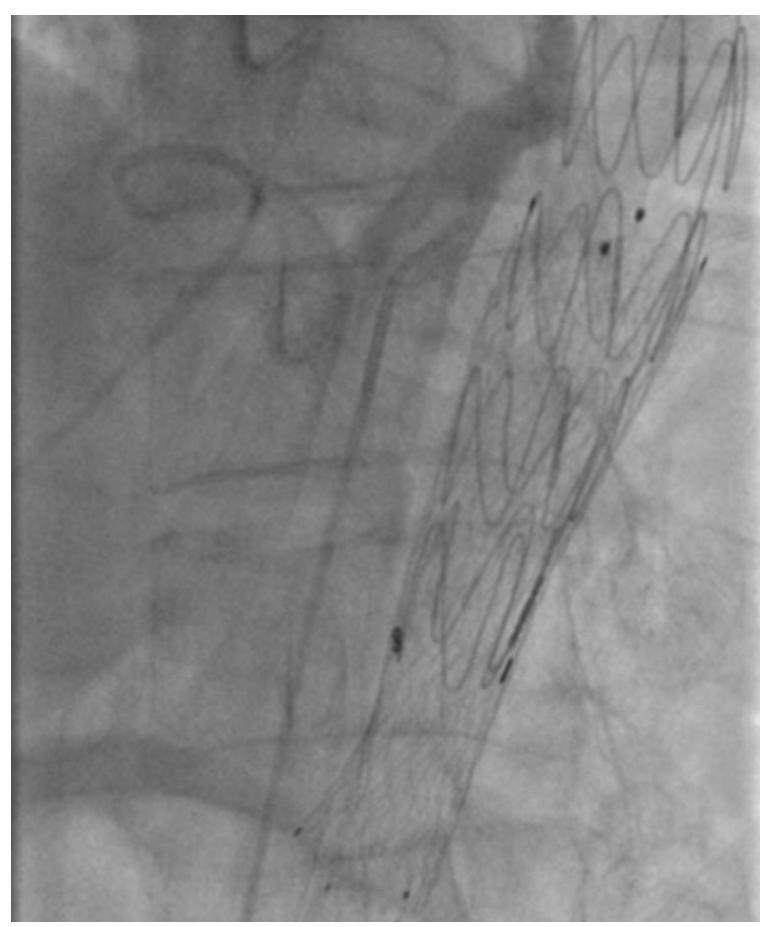

VIDEO 1. Intraoperative angiography showed TFLBs still played a role as the outflow tract to lead to persistent thoracic false lumen bloodstream after sealing the primary entry in TEVAR. Video available at: http://www. jtcvsonline.org/article/S0022-5223(17)31913-X/fulltext.

The rationale that complete thrombosis mainly occurs in the stent-graft-covered segment is based on sealing the proximal entry tear, reapproximating the flap to the aortic wall, and restricting motion of intimal flap throughout the cardiac cycle. ${ }^{1,37-39}$ However, as shown in the schematic drawing (Figure 4), the complete thrombosis of the stentgraft-covered false lumen is hampered by the presence of blood flow, which is maintained by outflow of patent TFLBs, including intercostal and bronchial arteries involved in dissection. In addition, these uncovered multiple downstream entry tears, which are common in DeBakey IIIb aortic dissections, provide adequate inflow for thoracic false lumen. Therefore, 1-stage transcatheter arterial embolization of selective TFLBs with microcoils is a potential approach to facilitate thoracic aortic false lumen remodeling of the stent-graft-covered segment.

In the current study, the preoperative TFLBs of the entire thoracic aortic segment, rather than the corresponding segment along the stent graft, were counted as parameters for analysis, based on the anatomic characteristics of the TFLBs and the patency of the blood flow pathway. First, the bloodstream in the false lumen of the stent-graftcovered segment was the result of the cumulative effects of the TFLBs as the outflow tracts of the false lumen of the thoracic aorta. The results of this analysis are supported by the results of the multivariate Cox regression, which demonstrated that the incidence of TAES increased 


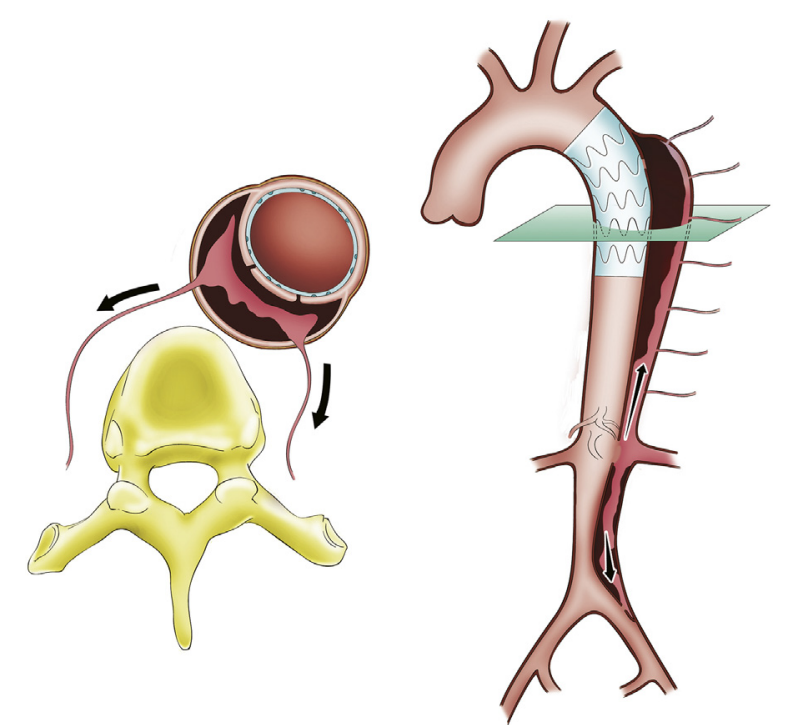

FIGURE 4. Schematic drawing demonstrates that the TFLBs as the outflow tract have an effect on perfusion of thoracic aortic false lumen along the stent graft after covering the proximal entry.

significantly with increases in preoperative TFLBs. Anatomically, the thoracic aortic branch arteries are small in diameter but large in quantity. As Poiseuille's law shows, the volumetric flow rate through a vessel is proportional to the fourth power of the vessel radius when the pressure drop and vessel length are constant. Thus, an increase in the number of TFLBs results in an exponential increase in the blood flow rate. Second, the thoracic aortic branch vessels, especially the posterior intercostal arteries, are continuously distributed along the descending thoracic aorta. The TFLBs distal to the stent graft play a role not only in increasing the blood flow rate in the thoracic aortic false lumen but also in maintaining the patency of the blood flow pathway. These findings also indicate that post-TEVAR thoracic aortic false lumen perfusion can be prevented by obstructing the false lumen, even in the portion of the aorta distal to the stent graft. A similar treatment strategy, known as the "cork in the bottleneck," was applied to treat chronic aortic dissection in 2003 by Loubert and colleagues. ${ }^{40}$

\section{Study Limitations}

This study had several limitations that must be considered when interpreting the data. First, this was a single-center retrospective observational study with a relatively small number of patients. Second, deaths before the TAES observed may lead to potential bias. Although all results in sensitivity analyses, as well as competing risk regressions, demonstrated that the 2 deaths did not bias the results significantly, studies in a larger size are still helpful to further verify the study results. Third, the diametric measurement at a fixed anatomic level may not remain the most clinically relevant because it cannot provide the threshold for a secondary intervention directly. However, it is a more sensitive way to quantify structural changes in the aorta over time. Fourth, the duration of the imaging follow-up period may be short for evaluation of aortic enlargement. However, previous observational studies have confirmed that the risk of false lumen dilation was higher in patients with partial, rather than complete, thrombosis of the false lumen. ${ }^{8-10,14,34,41}$ Moreover, as shown in the current study, aortic dissections with more preoperative TFLBs were more inclined to develop partially thrombosed false lumens over time than dissections with fewer preoperative TFLBs. Therefore, extending follow-up times would have likely further strengthened the observed association between TFLBs and TAES. Fifth, our hypothesis that TFLBs result in retrograde flow and partial thrombosis of the false lumen was based on persistent patent postoperative TFLBs. The number of preoperative TFLBs is not equivalent to postoperative TFLBs because some TFLBs may close spontaneously after successful endografting (Figure 1,D). We cannot accurately quantify the numbers of patent postoperative TFLBs even when using delayed contrast thin-cut imaging because the postoperative false lumen thrombosis impeded rapid distribution of contrast material in thoracic false lumen, leading to unsatisfied opacification of thoracic small branches on CTA images. The predictive value of preoperative TFLBs can be explained by that patients with more preoperative TFLBs also had more postoperative TFLBs. Future studies using intravascular ultrasonography and 4-dimensional phase contrast magnetic resonance imaging, which allow the hemodynamic characteristics of the aortic remolding process to be visualized and quantified, will better elucidate the association between TFLBs and TAES after TEVAR. ${ }^{42}$

\section{CONCLUSIONS}

Preoperative TFLBs are a morphologic predictor of TAES in DeBakey IIIb aortic dissection after TEVAR. Thoracic aortic false lumen remodeling of the stent-graftcovered segment consisting of diameter regression and complete thrombosis occurs more often in DeBakey IIIb aortic dissection with preoperative TFLBs less than 8 compared with preoperative TFLBs 8 or greater. Our findings may facilitate the identification of patients at higher risk of TAES using imaging scan before performing TEVAR.

\section{Conflict of Interest Statement}

Authors have nothing to disclose with regard to commercial support.

The authors thank Dr Ying Qi Wei (Beijing Center for Diseases Prevention and Control, China) for the statistical support during the development of this article. 


\section{References}

1. Dake MD, Kato N, Mitchell RS, Semba CP, Razavi MK, Shimono T, et al. Endovascular stent-graft placement for the treatment of acute aortic dissection. $N$ Engl J Med. 1999;340:1546-52.

2. Bavaria JE, Appoo JJ, Makaroun MS, Verter J, Yu ZF, Mitchell RS. Endovascular stent grafting versus open surgical repair of descending thoracic aortic aneurysms in low-risk patients: a multicenter comparative trial. J Thorac Cardiovasc Surg. 2007; 133:369-77.

3. Beregi JP, Haulon S, Otal P, Thony F, Bartoli JM, Crochet D, et al. Endovascular treatment of acute complications associated with aortic dissection: midterm results from a multicenter study. J Endovasc Ther. 2003;10:486-93.

4. Nienaber CA, Kische S, Rousseau H, Eggebrecht H, Rehders TC, Kundt G, et al. Endovascular repair of type $\mathrm{B}$ aortic dissection: long-term results of the randomized investigation of stent grafts in aortic dissection trial. Circ Cardiovasc Interv. 2013;6:407-16.

5. Brunkwall J, Kasprzak P, Verhoeven E, Heijmen R, Taylor P, Alric P, et al. Endovascular repair of acute uncomplicated aortic type B dissection promotes aortic remodelling: 1 year results of the ADSORB trial. Eur J Vasc Endovasc Surg. 2014;48:285-91.

6. Ziza V, Canaud L, Molinari N, Branchereau P, Marty-Ane C, Alric P. Thoracic endovascular aortic repair: a single center's 15-year experience. J Thorac Cardiovasc Surg. 2016;151:1595-603.e1597.

7. Ehrlich MP, Rousseau H, Heijmen R, Piquet P, Beregi JP, Nienaber CA, et al. Midterm results after endovascular treatment of acute, complicated type B aortic dissection: the Talent Thoracic Registry. J Thorac Cardiovasc Surg. 2013;145: 159-65.

8. Conrad MF, Crawford RS, Kwolek CJ, Brewster DC, Brady TJ, Cambria RP. Aortic remodeling after endovascular repair of acute complicated type B aortic dissection. J Vasc Surg. 2009;50:510-7.

9. Rodriguez JA, Olsen DM, Lucas L, Wheatley G, Ramaiah V, Diethrich EB. Aortic remodeling after endografting of thoracoabdominal aortic dissection. $J$ Vasc Surg. 2008;47:1188-94

10. Sayer D, Bratby M, Brooks M, Loftus I, Morgan R, Thompson M. Aortic morphology following endovascular repair of acute and chronic type B aortic dissection: implications for management. Eur J Vasc Endovasc Surg. 2008;36: 522-9.

11. Eggebrecht H, Nienaber CA, Neuhauser M, Baumgart D, Kische S, Schmermund A, et al. Endovascular stent-graft placement in aortic dissection: a meta-analysis. Eur Heart J. 2006;27:489-98.

12. VIRTUE Registry Investigators. Mid-term outcomes and aortic remodelling after thoracic endovascular repair for acute, subacute, and chronic aortic dissection: the VIRTUE Registry. Eur J Vasc Endovasc Surg. 2014;48:363-71.

13. Resch TA, Delle M, Falkenberg M, Ivancev K, Konrad P, Larzon T, et al. Remodeling of the thoracic aorta after stent grafting of type B dissection: a Swedish multicenter study. J Cardiovasc Surg (Torino). 2006;47:503-8.

14. Tsai TT, Evangelista A, Nienaber CA, Myrmel T, Meinhardt G, Cooper JV, et al Partial thrombosis of the false lumen in patients with acute type B aortic dissection. N Engl J Med. 2007;357:349-59.

15. Evangelista A, Salas A, Ribera A, Ferreira-Gonzalez I, Cuellar H, Pineda V, et al. Long-term outcome of aortic dissection with patent false lumen: predictive role of entry tear size and location. Circulation. 2012;125:3133-41.

16. Qin YL, Deng G, Li TX, Jing RW, Teng GJ. Risk factors of incomplete thrombosis in the false lumen after endovascular treatment of extensive acute type B aortic dissection. J Vasc Surg. 2012;56:1232-8.

17. Tolenaar JL, Kern JA, Jonker FH, Cherry KJ, Tracci MC, Angle JF, et al. Predictors of false lumen thrombosis in type B aortic dissection treated with TEVAR. Ann Cardiothorac Surg. 2014;3:255-63.

18. Batchelor GK. An introduction to fluid dynamics. Am Math Month. 1967;76:957.

19. Kolbel T, Tsilimparis N, Wipper S, Larena-Avellaneda A, Diener H, Carpenter SW, et al. TEVAR for chronic aortic dissection - is covering the primary entry tear enough? J Cardiovasc Surg (Torino). 2014;55:519-27.

20. Song SW, Kim TH, Lim SH, Lee KH, Yoo KJ, Cho BK. Prognostic factors for aorta remodeling after thoracic endovascular aortic repair of complicated chronic DeBakey IIIb aneurysms. J Thorac Cardiovasc Surg. 2014;148:925-32. 933.e921.

21. Grabenwoger M, Alfonso F, Bachet J, Bonser R, Czerny M, Eggebrecht H, et al. Thoracic Endovascular Aortic Repair (TEVAR) for the treatment of aortic diseases: a position statement from the European Association for Cardio-Thoracic
Surgery (EACTS) and the European Society of Cardiology (ESC), in collaboration with the European Association of Percutaneous Cardiovascular Interventions (EAPCI). Eur Heart J. 2012;33:1558-63.

22. Erbel R, Aboyans V, Boileau C, Bossone E, Di Bartolomeo R, Eggebrecht $\mathrm{H}$, et al. [2014 ESC Guidelines on the diagnosis and treatment of aortic diseases] Kardiol Pol. 2014;72:1169-252.

23. Jia X, Guo W, Li TX, Guan S, Yang RM, Liu XP, et al. The results of stent graft versus medication therapy for chronic type B dissection. J Vasc Surg. 2013;57: 406-14.

24. Feng J, Lu Q, Zhao Z, Bao J, Feng X, Qu L, et al. Restrictive bare stent for prevention of stent graft-induced distal redissection after thoracic endovascular aortic repair for type B aortic dissection. J Vasc Surg. 2013;57:44s-52s.

25. Kirby JM, Jhaveri KS, Kachura JR. Computed tomography angiography in abdominal aortic endoleaks: what is the optimal protocol? Can Assoc Radiol J 2007:58:264-71.

26. Von Tengg-Kobligk H, Weber TF, Rengier F, Kotelis D, Geisbusch P, Bockler D, et al. Imaging modalities for the thoracic aorta. J Cardiovasc Surg (Torino). 2008; 49:429-47.

27. Bland JM, Altman DG. Statistical methods for assessing agreement between two methods of clinical measurement. Lancet. 1986;1:307-10.

28. Manning BJ, Dias N, Manno M, Ohrlander T, Malina M, Sonesson B, et al. Endovascular treatment of acute complicated type B dissection: morphological changes at midterm follow-up. J Endovasc Ther. 2009;16:466-74.

29. Lombardi JV, Cambria RP, Nienaber CA, Chiesa R, Mossop P, Haulon S, et al Aortic remodeling after endovascular treatment of complicated type B aortic dissection with the use of a composite device design. J Vasc Surg. 2014;59: 1544-54.

30. Diehm N, Vermassen F, van Sambeek MR. Standardized definitions and clinical endpoints in trials investigating endovascular repair of aortic dissections. Eur $J$ Vasc Endovasc Surg. 2013;46:645-50.

31. Lee PH, Burstyn I. Identification of confounder in epidemiologic data contami nated by measurement error in covariates. BMC Med Res Methodol. 2016;16:54.

32. Fine JP, Gray RJ. A proportional hazards model for the subdistribution of a competing risk. J Am Stat Assoc. 1999;94:496-509.

33. Scrucca L, Santucci A, Aversa F. Regression modeling of competing risk using R: an in depth guide for clinicians. Bone Marrow Transplant. 2010;45:1388-95.

34. Watanabe Y, Shimamura K, Yoshida T, Daimon T, Shirakawa Y, Torikai K, et al. Aortic remodeling as a prognostic factor for late aortic events after thoracic en dovascular aortic repair in type B aortic dissection with patent false lumen. $J$ Endovasc Ther. 2014;21:517-25.

35. Luebke T, Brunkwall J. Type B Aortic dissection: a review of prognostic factors and meta-analysis of treatment options. Aorta (Stamford). 2014;2:265-78

36. Li D, Ye L, He Y, Cao X, Liu J, Zhong W, et al. False lumen status in patients with acute aortic dissection: a systematic review and meta-analysis. J Am Heart Assoc 2016;5:e03172.

37. Hofferberth SC, Nixon IK, Boston RC, McLachlan CS, Mossop PJ. Stent-assisted balloon-induced intimal disruption and relamination in aortic dissection repair: the STABILISE concept. J Thorac Cardiovasc Surg. 2014;147: 1240-5.

38. Nienaber CA, Kische S, Zeller T, Rehders TC, Schneider H, Lorenzen B, et al Provisional extension to induce complete attachment after stent-graft placement in type B aortic dissection: the PETTICOAT concept. J Endovasc Ther. 2006;13: 738-46.

39. Nauta FJ, van Bogerijen GH, Trentin C, Conti M, Auricchio F, Moll FL, et al Impact of thoracic endovascular aortic repair on pulsatile circumferential and longitudinal strain in patients with aneurysm. J Endovasc Ther. 2017;24:281-9.

40. Loubert MC, van der Hulst VP, De Vries C, Bloemendaal K, Vahl AC. How to exclude the dilated false lumen in patients after a type B aortic dissection? The cork in the bottleneck. J Endovasc Ther. 2003;10:244-8.

41. Trimarchi S, Tolenaar JL, Jonker FH, Murray B, Tsai TT, Eagle KA, et al. Importance of false lumen thrombosis in type B aortic dissection prognosis. J Thorac Cardiovasc Surg. 2013;145:S208-12.

42. Clough R, Taylor P. Future imaging techniques in aortic pathologies and clinica implications. J Cardiovasc Surg (Torino). 2013;54:15-9.

Key Words: aortic dissection, TEVAR, aortic remodeling, thoracic false lumen branches 
Method: NNE, Time $=132$, AUC $=0.864$

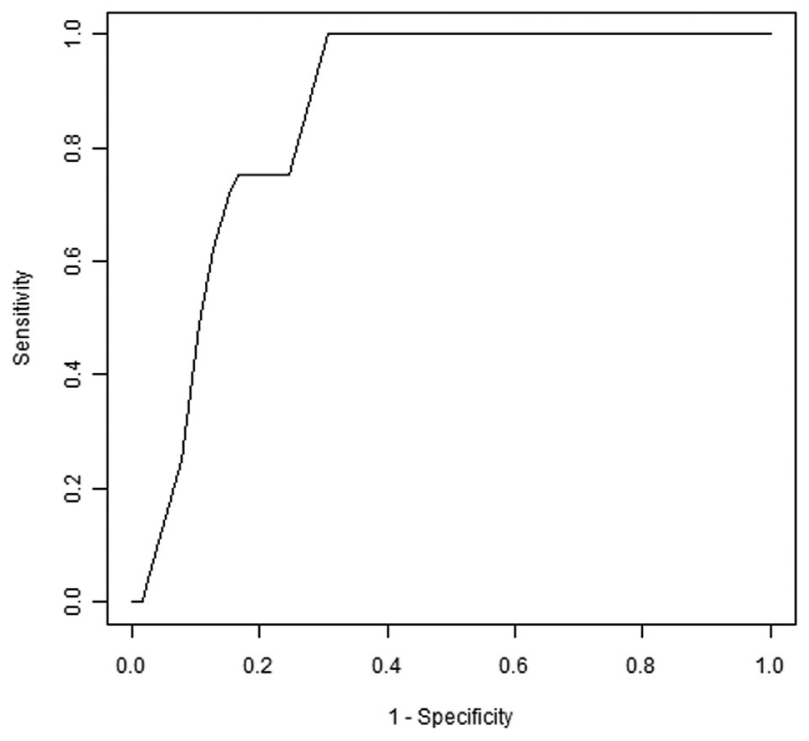

FIGURE E1. Receiver operating characteristic curve for TAES and preoperative TFLBs. The optimal cutoff value of preoperative TFLBs for prediction of TAES during follow-up was 8 with $100.0 \%$ sensitivity and $69.4 \%$ specificity. The area under the curve was 0.864 . NNE, Nearest neighbor estimation; $A U C$, area under the curve. 
TABLE E1. Comparison of the included and excluded patients' baseline characteristics

\begin{tabular}{|c|c|c|c|}
\hline Variables & $\begin{array}{c}\text { Included patients } \\
\qquad N=67\end{array}$ & $\begin{array}{l}\text { Excluded patients } \\
\qquad \mathbf{N}=19\end{array}$ & $P$ value \\
\hline \multicolumn{4}{|l|}{ Demographics } \\
\hline Female, \% (n) & $14.9(10)$ & $26.3(5)$ & .25 \\
\hline Age, y & $51.2 \pm 10.2$ & $55.2 \pm 9.7$ & .11 \\
\hline BMI, $\mathrm{kg} / \mathrm{m}^{2}$ & $27.0 \pm 4.2$ & $25.5 \pm 4.4$ & .18 \\
\hline Hypertension & $92.5(62)$ & $89.5(17)$ & .65 \\
\hline Diabetes & $1.5(1)$ & $0(0)$ & .99 \\
\hline Hyperlipidemia & $11.9(8)$ & $5.3(1)$ & .68 \\
\hline Carotid atherosclerosis & $11.9(8)$ & $10.5(2)$ & .99 \\
\hline Coronary artery disease & $11.9(8)$ & $10.5(2)$ & .99 \\
\hline Smoking & $52.2(35)$ & $52.6(10)$ & .99 \\
\hline Renal insufficiency & $9.0(6)$ & $5.63(1)$ & .99 \\
\hline Chronic obstructive pulmonary disease & $4.5(3)$ & $5.3(1)$ & .99 \\
\hline \multicolumn{4}{|l|}{ Dissection characteristics } \\
\hline Acute dissection, $\%(\mathrm{n})$ & $74.6(50)$ & $89.5(17)$ & .22 \\
\hline Complicated dissection, \% (n) & $62.7(42)$ & $68.4(13)$ & .65 \\
\hline \multicolumn{4}{|l|}{ Preoperative morphologic characteristics } \\
\hline Maximum aortic diameter, (n) & $37.4 \pm 5.4(67)$ & $37.2 \pm 6.5(18)$ & .92 \\
\hline True lumen diameter, (n) & $17.3 \pm 5.4(67)$ & $17.7 \pm 4.1(18)$ & .74 \\
\hline False lumen diameter, (n) & $18.5 \pm 6.4(67)$ & $18.0 \pm 5.9(18)$ & .75 \\
\hline $\mathrm{L} / \mathrm{Hi},(\mathrm{n})$ & $0.25 \pm 0.05(67)$ & $0.25 \pm 0.04(18)$ & .76 \\
\hline No. of TFLBs (n) & $7(0-20)(67)$ & $6(0-14)(18)$ & .40 \\
\hline No. of involved visceral arteries (n) & $1(0,3)(67)$ & $0(0,2)(18)$ & .43 \\
\hline No. of abdominal false lumen branches (n) & $5(0-9)(67)$ & $5(0-10)(18)$ & .65 \\
\hline False lumen status of thoracic aorta, $\%(n / N)$ & & & .76 \\
\hline Partially thrombosed & $23.9(16 / 67)$ & $27.8(5 / 18)$ & \\
\hline Patent & $76.1(51 / 67)$ & $72.2(13 / 18)$ & \\
\hline False lumen status of abdominal aorta, $\%(\mathrm{n} / \mathrm{N})$ & & & .71 \\
\hline Completely thrombosed & $6.0(4 / 67)$ & $11.1(2 / 18)$ & \\
\hline Partially thrombosed & $14.9(10 / 67)$ & $16.7(3 / 18)$ & \\
\hline Patent & $79.1(53 / 67)$ & $72.2(13 / 18)$ & \\
\hline Fenestration in iliac artery, $\%(\mathrm{n} / \mathrm{N})$ & & & .60 \\
\hline No & $47.8(32 / 67)$ & $55.6(10 / 18)$ & \\
\hline Yes & $52.2(35 / 67)$ & $44.4(8 / 18)$ & \\
\hline \multicolumn{4}{|l|}{ Devices implanted, \% (n) } \\
\hline Stent-graft No. & & & 1.00 \\
\hline 1 stent-graft & $95.5(64)$ & $94.7(18)$ & \\
\hline 2 stent-grafts & $4.5(3)$ & $5.3(1)$ & \\
\hline Use of restrictive bare stent & $10.4(7)$ & $21.1(4)$ & .25 \\
\hline Additional bare mental stent & $4.5(3)$ & $0(0)$ & 1.00 \\
\hline
\end{tabular}

Values are mean \pm standard deviation, mean (range), unless otherwise noted. BMI, Body mass index; $L / H i$, length of dissected aorta and height index; $T F L B$, thoracic false lumen branch. 
TABLE E2. Univariable Cox regression analyses for associations between each variable and thoracic aortic enlargement along the stent grafts

\begin{tabular}{|c|c|c|}
\hline & HR $(95 \%$ CI $)$ & $P$ value \\
\hline Female (vs male) & $1.06(0.12-9.12)$ & .95 \\
\hline Age (for 1-y increase) & $0.98(0.91-1.06)$ & .58 \\
\hline BMI (for 1 increase) & $1.02(0.85-1.21)$ & .86 \\
\hline Hyperlipidemia & $1.22(0.14-10.51)$ & .85 \\
\hline Smoke & $0.42(0.08-2.31)$ & .32 \\
\hline Acute dissection (vs chronic) & $0.33(0.07-1.64)$ & .17 \\
\hline Complicated dissection (vs uncomplicated) & $1.25(0.23-6.82)$ & .80 \\
\hline Maximum aortic diameter (for 1-mm increase) & $1.00(0.85-1.16)$ & .96 \\
\hline True lumen diameter (for 1-mm increase) & $1.09(0.93-1.27)$ & .29 \\
\hline False lumen diameter (for 1-mm increase) & $0.94(0.82-1.08)$ & .37 \\
\hline $\mathrm{L} / \mathrm{Hi}$ (for 1 increase) & $6.26(0.79-49.78)$ & .09 \\
\hline Preoperative TFLBs (for 1 increase) & $1.23(1.05-1.45)$ & .01 \\
\hline Preoperative abdominal false lumen branches (for 1 increase) & $1.03(0.79-1.34)$ & .83 \\
\hline Involved visceral arteries (for 1 increase) & $0.35(0.07-1.70)$ & .19 \\
\hline Fenestration in iliac artery (yes vs no) & $1.67(0.31-9.14)$ & .55 \\
\hline The length of stent-graft coverage (for 1-mm increase) & $0.99(0.95-1.02)$ & .52 \\
\hline Distal SINE (yes vs no) & $3.19(0.57-17.80)$ & .19 \\
\hline
\end{tabular}

HR, Hazard ratio; $C I$, confidence interval; $B M I$, body mass index; $L / H i$, length of dissected aorta and height index; TFLB, thoracic false lumen branches; SINE, stent-graftinduced new reentry. 